\title{
Construtos de incapacidade presentes na Pesquisa Nacional por Amostra de Domicílio (PNAD): uma análise baseada na Classificação Internacional de Funcionalidade, Incapacidade e Saúde (CIF)
}

\author{
Luciana Castaneda* \\ Shamyr Sulyvan de Castro* \\ Ligia Bahia ${ }^{\star \star x}$
}

O objetivo deste artigo é identificar construtos relacionados à incapacidade presentes na Pesquisa Nacional por Amostra de Domicílios (PNAD), tendo como ferramenta teórica a Classificação Internacional de Funcionalidade, Incapacidade e Saúde (CIF). Trata-se de estudo metodológico do tipo descritivo. A primeira etapa da pesquisa compreendeu a identificação dos blocos integrantes do instrumento de coleta que continham conteúdos referentes a funcionalidade, incapacidade e deficiência. Posteriormente, foram extraídos os conteúdos significativos e codificados para as categorias da CIF. Verificou-se que os domínios mais frequentes foram os relacionados a atividades e participação, seguidos pelos domínios de funções do corpo. Apenas um conteúdo relacionou-se aos fatores ambientais. 0 estudo mostrou que a CIF é uma ferramenta com grande aplicabilidade nos sistemas de informação de dados primários. Por sua linguagem universal e perspectiva biopsicossocial, a classificação deve ser mais explorada no campo da epidemiologia.

Palavras-chave: Inquéritos epidemiológicos. Classificação Internacional de Funcionalidade, Incapacidade e Saúde. Coleta de dados. Sistemas de Informação. Epidemiologia.

\footnotetext{
* Instituto Brasileiro de Medicina de Reabilitação - Laureate Universities, Rio de Janeiro-RJ, Brasil (lucianacastaneda@ yahoo.com.br).

** Instituto de Ciências da Saúde (ICS), Universidade Federal do Triângulo Mineiro (UFTM), Uberaba-MG, Brasil (shamyr@ fisioterapia.uftm.edu.br).

*** Instituto de Estudos em Saúde Coletiva da Universidade Federal do Rio de Janeiro, Rio de Janeiro-RJ, Brasil (ligiabahia@ terra.com.br).
} 


\section{Introdução}

É amplamente reconhecida a importância de inquéritos populacionais como fontes primárias de dados sobre saúde, que são úteis para estudos de desigualdades no estado de saúde, no acesso e uso de serviços e no gasto privado. Esses estudos têm a capacidade de fornecer informações pertinentes ao perfil de saúde e aos fatores de risco ou de proteção em determinada população, podendo ainda ter atualização periódica e cobrir distintas regiões geográficas. Além disso, é possível correlacionar dados de problemas de saúde com as condições socioeconômicas e, assim, dimensionar a iniquidade em saúde persistente no quadro nacional (VIACAVA et al., 2006). A necessidade de conhecer a realidade epidemiológica reafirma o papel dos inquéritos domiciliares de saúde como ferramenta ideal para a geração dos dados, por meio de estudos transversais com periodicidade previamente definida, com a possibilidade também de realização em diferentes períodos, sendo possível avaliar em uma perspectiva longitudinal e temporal o efeito de intervenções, tais como as políticas que visam a promoção da saúde, e comparar os diferentes padrões de polarização epidemiológica (WALDMAN, 2008).

Desde o início de sua história, o Instituto Brasileiro de Geografia Estatística (IBGE) foi responsável pela elaboração e produção de uma série de pesquisas cujo conteúdo foi bastante significativo para a produção de informações sobre as características demográficas e socioeconômicas da população brasileira. Uma das ferramentas utilizadas pelo Instituto para a produção das informações é a Pesquisa Nacional por Amostra de Domicílios (PNAD) (OLIVEIRA; SIMÕES, 2005).

As medidas de avaliação funcional desenvolvidas vêm sendo criticadas por serem descoordenadas. Uma razão para isso é a falta de uma ferramenta teórica e conceitual clara, o que, da perspectiva dos inquéritos domiciliares de saúde, dificulta a comparação de resultados (RATY et al., 2003). A aferição da ocorrência de deficiência em grupos populacionais tem sido alvo de discussão acadêmica (McDERMOTT; TURLE, 2011; ALTMAN, 2011) e até mesmo são apontadas possíveis distorções nos números (ALTMAN; GULLEY, 2009). Esses problemas podem decorrer das dificuldades teóricas em torno do termo deficiência. Visando unificar a linguagem dos processos de funcionalidade e incapacidade, a Organização Mundial de Saúde publicou, em 2001, a Classificação Internacional de Funcionalidade (OPAS, 2003), baseada em uma abordagem biopsicossocial, que constitui uma síntese das abordagens médica e social do processo de incapacidade, em que cada dimensão desse processo (funções e estruturas corporais; atividades e participação) é conceituada como uma interação dinâmica entre os fatores intrínsecos ao indivíduo e o seu ambiente físico e social (fatores ambientais). A informação da CIF é organizada por meio de dois componentes. A parte 1 refere-se à funcionalidade e incapacidade e é representada pelas categorias funções do corpo - entendidas como funções fisiológicas e estruturas do corpo definidas como estruturas anatômicas - e atividades e participação, sendo a primeira categoria conceituada como a execução de uma tarefa ou ação por um indivíduo e a segunda como o envolvimento em uma situação de vida diária. A parte 2 trata dos fatores contextuais e contém os fatores 
ambientais, que compõem o ambiente físico, social e de atitude no qual as pessoas vivem e conduzem sua vida, e os fatores pessoais, que fazem parte do histórico particular da vida e do estilo de vida de um indivíduo, englobando características que não são parte de uma condição de saúde (WHO, 2001, p. 18, 19 e 29).

É interessante mencionar que esforços no sentido de discutir e melhorar a forma de coleta de dados por inquéritos populacionais já foram relatados no Brasil. Em um momento prévio à publicação da CIF, modelos conceituais que incorporavam atividades e limitações já foram testados, reforçando a necessidade de inclusão desses conceitos nos instrumentos de coleta (BERCOVICH, 2006). Dando prosseguimento a esse esforço, o Censo Demográfico de 2000 trouxe novas abordagens da deficiência, com o objetivo de superar dificuldades conceituais, incorporando uma compreensão ampliada da deficiência em franca aproximação com o modelo proposto pela CIF (BRASIL, 2008, p. 12).

Considerando a relevância da PNAD como peça fundamental no sistema de informação de bases de dados primários em saúde no âmbito nacional, a identificação dos conteúdos presentes tanto no instrumento de coleta da pesquisa quanto na CIF evidenciará de que maneira o inquérito aborda a temática da deficiência e dos processos de funcionalidade e incapacidade, uma vez que tais domínios apresentam-se como importantes preditores de indicadores de qualidade de vida relacionada à saúde e possuem maior capacidade de predição do que indicadores baseados somente em diagnósticos clínicos. Com esse panorama, pode-se proceder à identificação das dimensões do processo incapacitante possivelmente não contempladas, bem como sugerir ajustes no questionário, podendo também complementar a avaliação com outros instrumentos que cubram outros componentes pertinentes e relevantes, já que estes são consagrados na literatura como bons preditores dos quadros de morbidade e mortalidade (FAYED et al., 2011). Assim, este estudo tem o objetivo de identificar os conteúdos comuns referentes a deficiência, incapacidade e funcionalidade presentes no instrumento de coleta da PNAD 2008 na CIF.

\section{Metodologia}

Trata-se de estudo metodológico, do tipo descritivo. O instrumento de coleta analisado foi o Suplemento Saúde da PNAD 2008, sendo que o estudo dividiu-se em três etapas dispostas na Figura 1.

Na primeira etapa, analisaram-se os blocos e seções do instrumento de coleta. $\mathrm{Na}$ segunda, foram extraídos os conteúdos significativos das questões das seções e blocos selecionados e, na terceira, relacionou-se a ligação do conteúdo significativo selecionado com a categoria da CIF mais precisa. Esta última etapa do processo foi executada por dois pesquisadores independentes, com conhecimento da CIF e da sua taxonomia e experiência e treinamento no processo de codificação, ${ }^{1}$ segundo regras já propostas e publicadas (CIEZA

\footnotetext{
${ }^{1}$ Os dois pesquisadores que trabalharam de forma independente nesta fase foram um fisioterapeuta, mestre em saúde pública, com experiência na aplicação da CIF em estudos epidemiológicos, e outro fisioterapeuta, doutor em saúde coletiva, com treinamento no Brasil sobre a aplicação da CIF e estágio de doutorado-sanduíche de 12 meses no ICF-Research Branch (http://www.icf-research-branch.org/), onde passou por treinamento em aplicação da CIF nas diversas áreas da saúde.
} 
et. al., 2005). Ao final, foram comparados os resultados encontrados pelos codificadores independentes e resolvidas as discordâncias por consenso entre ambos.

Para a análise estatística descritiva, utilizaram-se medidas como números puros, porcentagens e frequências simples. O grau de concordância entre os dois profissionais que realizaram o processo de ligação foi calculado por meio do Coeficiente de Kappa simples e do intervalo de confiança (95\%). A escolha pelo grau de concordância entre dois avaliadores mantém a tendência da literatura nesse tipo de metodologia de ligação de instrumentos de coleta de dados com a CIF (KAHAN; AMATYA, 2009; GEYH et al., 2007; SCHEPERS et al., 2007). Além disso, o Coeficiente de Kappa é uma medida de concordância interobservador, que mede o nível de concordância retirando o efeito do acaso. Seus valores variam de 0 a 1, sendo que 1 indica concordância máxima e 0 nenhuma concordância (CALLEGARI-JACQUES, 2003). O software utilizado foi o SPSS (versão 7.0).

FIGURA 1

Fluxograma das etapas realizadas na metodologia do trabalho

\section{Suplemento Saúde da PNAD 2008}

1a etapa: Análise dos blocos e seções do instrumento de coleta

Blocos e seções selecionadas:

a. Saúde dos moradores - morbidade

b. Mobilidade física e fatores de risco à saúde dos moradores de 14 anos ou mais de idade

2ª etapa: Extração dos conteúdos significativos dos módulos selecionados

3a etapa: Ligação dos conteúdos significativos dos módulos selecionados com a CIF

Foram selecionados o bloco de saúde dos moradores (seção de morbidade) e o bloco sobre mobilidade física e fatores de risco à saúde dos moradores de 14 anos ou mais de idade (todas as seções). O processo de ligação entre os questionários da PNAD e a CIF baseou-se em metodologia amplamente difundida na literatura (GLASSEL et al., 2011; KAHAN; AMATYA, 2009; GEYH et al., 2007; SCHEPERS et al., 2007), proposta por Cieza et al. (2005). Os autores sugeriram oito regras para ligação entre as medidas de resultado (medidas clínicas, do estado de saúde e técnicas) e a CIF e mais cinco regras adicionais. As regras específicas determinam que todos os conceitos significantes devem ser considerados antes de se realizar a ligação com as categorias da CIF e que as opções de resposta, quando contenham conceitos relevantes, sejam incluídas. No caso de o conceito de algum item conter exemplos, estes também devem ser ligados. Tais regras foram desenvolvidas com base na experiência acumulada durante o processo de ligação de centenas de documentos de medidas clínicas e do estado de saúde realizadas desde a publicação da CIF em 2001. 


\section{Resultados}

Em relação à seção de morbidade do bloco sobre saúde dos moradores, das 18 perguntas foram extraídos 41 conteúdos significativos. Já para o bloco sobre mobilidade física e fatores de risco à saúde dos moradores de 14 anos ou mais de idade, das 16 perguntas foram coletados 45 conteúdos, conforme disposto na Figura 2.

\section{FIGURA 2}

Número de conteúdos significativos identificados na PNAD 2008 e suas distribuições entre os componentes da CIF

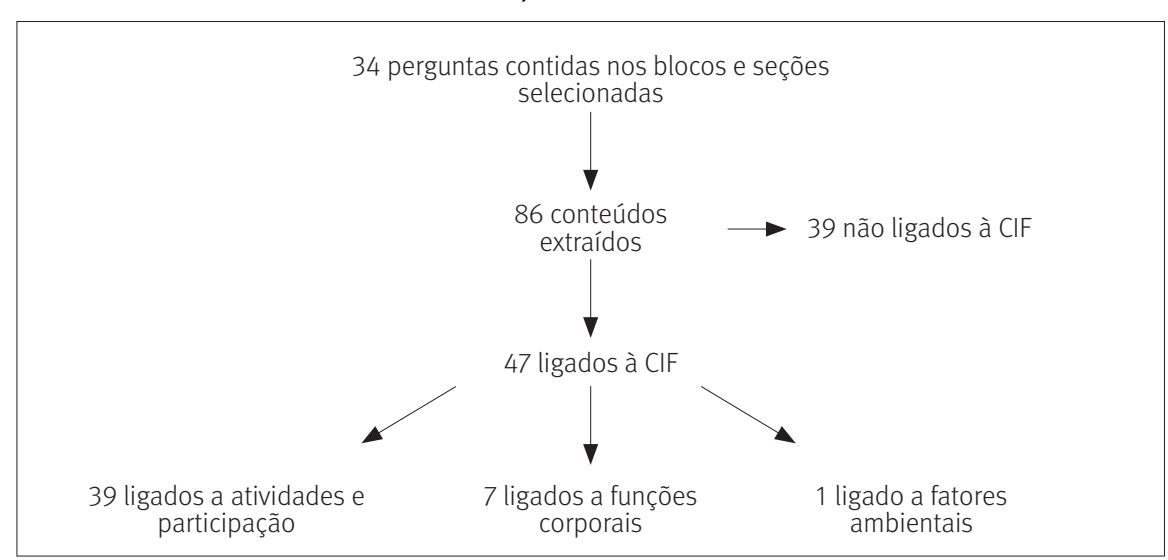

Dos 86 conteúdos significativos extraídos do questionário do Suplemento Saúde da PNAD 2008, 39 correspondem a categorias de atividades e participação, sete referem-se a funções corporais, um relaciona-se a fatores ambientais, 31 não são cobertos pela classificação, dois eram não definíveis em saúde mental, cinco não definíveis em atividades e participação e um não definível em saúde geral (Tabela 1). Os conteúdos não contemplados pela CIF dizem respeito a domínios do tempo, condições de saúde (tais como doenças, injúrias e lesões que são codificadas pela Classificação Internacional de Doenças - CID) e ainda fatores pessoais.

TABELA 1

Distribuição dos resultados da ligação dos módulos selecionados do questionário da PNAD 2008 com a CIF (morbidade e características de mobilidade física e fatores de risco à saúde dos moradores de 14 anos ou mais)

\begin{tabular}{lcc}
\hline \multicolumn{1}{c}{ Categorias } & N. abs. & \% \\
\hline Funções corporais & 7 & 8,1 \\
Atividades e participação & 39 & 45,3 \\
Fatores ambientais & 1 & 1,1 \\
Não definível - atividade e participação & 5 & 5,8 \\
Não definível - saúde mental & 2 & 2,3 \\
Não definível - saúde geral & 1 & 2,3 \\
Não coberto & 31 & 36,0 \\
Total & $\mathbf{8 6}$ & $\mathbf{1 0 0 , 0}$ \\
\hline
\end{tabular}

Fonte: IBGE. Pesquisa Nacional por Amostra de Domicílios - 2008; Classificação Internacional de Funcionalidade, Incapacidade e Saúde. 
Para o bloco de saúde dos moradores (seção de morbidade), dos 41 conteúdos significativos extraídos, três referem-se a atividades e participação, seis relacionam-se a funções corporais, 26 não são cobertos pela classificação, dois eram não definíveis em saúde mental, três não definíveis em atividades e participação e um não definível em saúde geral. Para a seção sobre características de mobilidade física e fatores de risco à saúde dos moradores de 14 anos ou mais de idade, dos 45 conteúdos, 36 referem-se a atividades e participação, um corresponde a fatores ambientais, um relaciona-se a funções corporais, dois não eram definíveis em atividades e participação e cinco não eram cobertos pelos domínios da CIF.

Em relação às análises de concordância, foram alcançados 64,7\% de concordância total (para as duas seções analisadas), 80,5\% para a seção do módulo de morbidade e 74,7\% para a seção de características de mobilidade física e fatores de risco à saúde dos moradores de 14 anos ou mais de idade. O Coeficiente de Kappa encontrado para a primeira seção foi de 0,78 (IC: 0,65 - 0,84) e, para a segunda, correspondeu a 0,69 (IC: 0,54-0,76). A concordância total foi de 0,59 (IC: 0,51-0,72).

\section{Discussão}

Em 1998, o Ministério da Saúde solicitou a realização de um inquérito sobre acesso e utilização de serviços de saúde. Com isso, foi inaugurada uma série histórica quinquenal de informação de base populacional sobre saúde e consumos de serviços, possibilitando o acompanhamento de aspectos relevantes da saúde da população brasileira e o monitoramento com distintos recortes geográficos e socioeconômicos, bem como a incorporação das informações de saúde pela sociedade e por diferentes áreas do governo, favorecendo assim as ações intersetoriais.

A estrutura da PNAD é contemplada por dois níveis informativos, sendo que o primeiro engloba dados referentes aos domicílios e o segundo traz informações sobre os indivíduos residentes (inclusive aqueles que estão ausentes por um período de até 12 meses). No nível do domicílio, são coletadas informações sobre características gerais, condições de ocupação, abastecimento de água, esgoto sanitário, iluminação elétrica, bens (telefones fixos, celulares, computador e acesso à Internet) e destino de lixo. Já no nível individual, são contempladas informações demográficas, cor ou raça, migração, características do trabalho e rendimento para maiores de dez anos de idade, características do trabalho infantil, fecundidade e escolaridade (TRAVASSOS et al., 2008).

Com a análise do instrumento de coleta do Suplemento Saúde da PNAD 2008, é possível contribuir para uma melhor compreensão dos conceitos de funcionalidade, incapacidade e qualidade de vida contemplados no instrumento. Ao fazer a ligação das medidas do estado de saúde presentes no questionário com a CIF, pode-se verificar a relação existente entre o conteúdo do instrumento e a classificação e, assim, esclarecer e elucidar seus conceitos, de acordo com a linguagem unificada proposta pela OMS. 
Em uma análise de três indicadores sobre a temática da incapacidade na PNAD 1998, verificou-se que a prevalência de incapacidade para abaixar-se, ajoelhar-se ou curvar-se nos idosos foi de 4,4\% (2,7\% para o sexo masculino e 5,7\% para o feminino). Já para as tarefas de alimentar-se, tomar banho ou ir ao banheiro, a prevalência foi de $2,0 \%$ (1,8\% para os homens e 2,2\% para as mulheres) e, para a tarefa de andar mais de um quilômetro, correspondeu a 6,2\% (4,2\% e 7,9\% para homens e mulheres, respectivamente). A escolha dos dois primeiros indicadores deveu-se ao fato de que, geralmente, essas tarefas são incluídas nos estudos epidemiológicos sobre o tema e também por se tratar de indicadores relacionados à perda da autonomia, enquanto o último indicador relaciona-se à capacidade do idoso de realizar atividades físicas de intensidade leve a moderada (LIMA-COSTA et al., 2005).

Costa (2008), ao analisar os dados sobre incapacidade no Suplemento Saúde da PNAD de 2003 , aponta que $21 \%$ do total de entrevistados relataram dificuldade para correr, levantar objetos pesados, praticar esportes ou trabalhos pesados (3,5\% não conseguiram realizar a tarefa), sendo esta a atividade mais comprometida. Em 2008, essas porcentagens mantiveram-se relativamente próximas às de 2003, com registros de 23,08\% e de 3,78\%, respectivamente (IBGE, 2008, p. 153). A limitação das atividades foi menor entre as pessoas de 14 a 39 anos e maior para aquelas de 60 anos e mais. Na faixa etária de 40 a 59 anos, a prevalência de incapacidade variou de $20 \%$ a 30\%. Em sua discussão, Costa aponta que os resultados para esta última faixa etária reafirmam a preocupação de outros autores com a elevada carga de morbidade que se origina na vida adulta e repercute negativamente na qualidade de vida dos idosos. O autor indica um possível viés que pode ter interferido nos resultados: a maior prevalência de incapacidade na população feminina pode ser influenciada pelo viés de sobrevivência seletiva (maior carga de mortalidade precoce no sexo masculino e estrutura envelhecida da população feminina). Nesse sentido, Parahyba e Veras (2008) assinalam que a questão "dificuldade para alimentar-se, ir ao banheiro ou tomar banho" pode conter um viés de informação em função das diferenças na acessibilidade das instalações sanitárias para os diferentes grupos, em um país heterogêneo como o Brasil. Além disso, em uma mesma pergunta estão contidas três atividades que exigem capacidades funcionais diferentes. No entanto, os autores apontam que os dados, embora apresentem certas limitações, possuem robustez e que, em relação às diferentes coortes (1998 e 2003), houve redução na perda de capacidade funcional dos idosos brasileiros, tendência que não teve continuidade segundo a pesquisa de 2008 (IBGE, 2008).

O presente estudo teve como limitações a baixa concordância interexaminador para a segunda seção analisada do instrumento de coleta da PNAD 2008. As perguntas dessa seção abordavam características de mobilidade física e prática de exercício da população com 14 anos ou mais. Essa limitação pode ser relacionada ao fato de os domínios de atividades e participação estarem contidos em um mesmo construto da CIF, o que pode demonstrar que esses dois componentes podem não ser perfeitamente discerníveis, ocasionando eventual dissenso na codificação. Houve um maior nível de concordância para os domínios de funções 
do corpo e estruturas do corpo, possivelmente pelo fato de esses domínios apresentarem definições mais claras e menos subjetivas, facilitando o processo de ligação pelos dois examinadores.

O fato de não existir nenhum estudo na literatura nacional com o mesmo tipo de metodologia (ligação da CIF com inquéritos de saúde de base populacional) limita a discussão externa no trabalho. Há na literatura nacional estudos envolvendo metodologia semelhante, mas com objetos distintos. Os estudos nacionais envolvem o processo de ligação da CIF com instrumentos de avaliação clínica (CASTANEDA; PLÁCIDO, 2009; CASTANEDA et al., 2011). Em um estudo internacional que realizou a ligação da CIF com diversos inquéritos europeus (conduzidos de 1998 a 2002), verificou-se que a maior parte dos questionários utilizados apresenta maior frequência de perguntas relacionadas a atividades e participação, seguidas por questões sobre funções corporais, com poucas perguntas que tratavam dos fatores ambientais (RATY et al., 2003). Os resultados encontrados nesse estudo vão ao encontro daqueles obtidos no presente trabalho.

Apenas um conteúdo significativo foi relacionado aos fatores ambientais. 0 ambiente é um dos seis componentes propostos pela CIF como parte do processo de funcionalidade e incapacidade e, dessa forma, se relaciona intimamente com os demais componentes (funções corporais, estruturas corporais, fatores pessoais, atividades e participação). Não contemplar os fatores ambientais no processo de coleta de dados sobre a incapacidade empobrece a análise dos quadros de morbidade na população. É sabido que pessoas com a mesma condição de saúde podem apresentar diferentes níveis de funcionalidade e incapacidade, ao passo que pessoas com diferentes condições de saúde podem experimentar o mesmo grau de funcionalidade e incapacidade. A OMS conceitua que estes fenômenos, além de multifatoriais, são influenciados pela condição de saúde, pelos recursos (econômicos e habilidade pessoais) e também pelo ambiente onde a pessoa está inserida. Versões futuras da PNAD poderiam incluir em seus instrumentos de coleta essa temática de modo mais consistente.

\section{Conclusão}

Os resultados apontam para um predomínio de domínios relacionados a atividades e participação, seguidos por domínios de funções do corpo. Observou-se, ainda, que somente um domínio relacionou-se aos fatores ambientais, o que indica que o ambiente é pouco contemplado no instrumento de coleta da PNAD.

Ao utilizar a CIF como uma ferramenta padrão para o embasamento dos instrumentos de coleta nos inquéritos, é possivel que se tenha uma diretriz em relação às perguntas contempladas, otimizando, assim, a comparação entre o mesmo inquérito em diferentes períodos. Os resultados encontrados podem servir como exemplo da utilização dos itens dos inquéritos para a comparação de instrumentos e questões e a sua ligação com as categorias da CIF, mas futuros estudos com outros inquéritos populacionais nacionais se fazem necessários para comparação dos resultados obtidos. 


\section{Referências}

ALTMAN, B. M. A reply to. The myth and reality of disability prevalence: measuring disability for research and service. Disability and Health, v. 4, n. 3, p. 198-209, 2011.

ALTMAN, B. M.; GULLEY, S. P. Convergence and divergence: differences in disability prevalence estimates in the United States and Canada based on four health survey instruments. Social Science Medicine, v. 9, n. 4, p. 543-552, 2009.

BERCOVICH, A. People with disabilities in Brazil: a look at 2000 Census results. In: ALTMAN, B. M.; BARNARTT, S. N. (Orgs.). International views on disability measures: moving toward comparative measurement. (Research in Social Science and Disability). New York: Elsevier Ltd., v. 4, 2006, p. 131-158.

BRASIL. Ministério da Saúde. Secretaria de Atenção à Saúde. Política Nacional de Saúde da Pessoa Portadora de Deficiência. Brasília: Editora do Ministério da Saúde, 2008.

CALLEGARI-JACQUES, S. M. Bioestatística. Princípios e aplicações. Porto Alegre: Artmed, 2003.

CASTANEDA, L.; CAMARGO, T.; PLÁCIDO, T. Ligação entre o M. D. Anderson Symptom Inventory-Head and Neck Module e a Classificação Internacional de Funcionalidade, para avaliação de pacientes com câncer de cabeça e pescoço. Revista Brasileira de Cancerologia, v. 57, n. 1, p. 49-55, 2011.

CASTANEDA, L.; PLACIDO, T. Ligação do King’s Heath Questionário com a Classificação Internacional de Funcionalidade, Incapacidade e Saúde, para avaliação de pacientes com incontinência urinária pós cirurgia oncológica ginecológica. Acta Fisiátrica, v. 17, n. 1, p. 18-21, 2009.

CIEZA, A.; GEYH, S.; CHATTERJU, S.; KOSTANKSEK, N.; USTUB, B.; STUCKI, G. ICF linking rules: an update bases on lessons learned. Journal of Rehabilitation Medicine, v. 37, n. 4, p. 212-218, 2005.

COSTA, A. J. Metodologias e indicadores da capacidade funcional: análise preliminar do Suplemento Saúde da Pesquisa Nacional por Amostra de Domicílios - PNAD Brasil, 2003. Ciência e Saúde Coletiva, v. 11, n. 4, p. 927-940, 2008.

FAYED, N.; CIEZA, A.; BICKENBACK, J. Linking health and health-related information to the ICF: a systematic review of the literature from 2001 to 2008. Disability and Rehabilitation, v. 33, n. 21-22, p. 1941-1951, 2011.

GEYH, S.; CIEZA, A.; KOLLERITS, B.; GRIMBY, G.; STUCKI, G. Content comparison of health-related quality of life measures used in stroke based on the international classification of functioning, disability and health (ICF): a systematic review. Quality of Life Research, v. 16, n. 5, p. 833-851, 2007.

GLASSEL, A.; FINGER, M. E.; CIEZA, A.; TREITER, C.; COENEN, M.; ESCORPIZO, R. Vocational rehabilitation from the client's perspective using the international classification of functioning, disability and health (ICF) as a reference. Journal of Occupational Rehabilitation, v. 21, n. 2, p. 167-178, 2011.

IBGE - Instituto Brasileiro de Geografia e Estatística. Pesquisa Nacional por Amostra de Domicílios. Um panorama da saúde no Brasil - Acesso e utilização dos serviços, condições de saúde e fatores de risco e proteção à saúde 2008. Rio de Janeiro, 2010. Disponivel em: 〈http://www.ibge.gov.br/home/ estatistica/populacao/panorama_saude_brasil_2003_2008/defaulttab_pdf_2.shtm>. Acesso em: 15 set. 2013.

KAHAN, F.; AMATYA, B. Use of the International Classification of Functioning, Disability and Health to describe patient-reported disability: a comparison of Guillain Barré syndrome with multiple sclerosis in a community cohort. Journal of Rehabilitation Medicine, v. 42, n. 8, p. 708-714, 2009.

LIMA-COSTA, M. F.; BARRETO, S. M.; GIATTI, L. Condições de saúde, capacidade funcional, uso de serviços de saúde e gastos com medicamentos da população idosa brasileira: um estudo descritivo baseado na Pesquisa Nacional por Amostra de Domicílios. Cadernos de Saúde Pública, v. 19, n. 3, p. 735-743, 2005. 
MCDERMOTT, S.; TURK, M. A. The myth and reality of disability prevalence: measuring disability for research and service. Disability and Health, v. 4, n. 1, p. 1-5, 2011.

OLIVEIRA, L. A.; SIMÕES, C. C. O IBGE e as pesquisas populacionais. Revista Brasileira de Estudos de População, v. 22, n. 20, p. 291-302, 2005.

OPAS - Organização Panamericana de Saúde; OMS - Organização Mundial da Saúde. CIF: Classificação Internacional de Funcionalidade, Incapacidade e Saúde. São Paulo: Edusp, 2003.

PARAYBHA, M. I.; VERAS, R. Diferencias sociodemográficos no declínio funcional em mobilidade física entre os idosos no Brasil. Ciência e Saúde Coletiva, v. 13, n. 4, p. 1257-1264, 2008.

RATY, S.; AROMAA, A.; KOPONEN, P. Measurement of physical functioning in comprehensive e national health surveys - ICF as a framework. Oakland: National Public Health Institute, 2003. Disponivel em: 〈http://www.euhsid.org/docs/HISHESphase2_sub4.pdf〉. Acesso em: 05 jan. 2011.

SCHEPERS, V. D.; KETELAAN, M.; VAN DE PORT, N.; VISSER-MERLY, J. M.; LINDEMANN, E. Comparing contents of functional outcome measures in stroke rehabilitation using the International Classification of Functioning, Disability and Health. Disability and Rehabilitation, v. 29, n. 3, p. 221-230, 2007.

TRAVASSOS, C.; VIACAVA, F., LAGUARDIA, J. Os Suplementos Saúde na Pesquisa Nacional por Amostra de Domicílios (PNAD) no Brasil. Revista Brasileira de Epidemiologia, v.11, n.1, p. 98-112, 2008.

VIACAVA, F.; DACHS, N.; TRAVASSOS, C. Os inquéritos domiciliares e o Sistema Nacional de Informações em Saúde. Ciência e Saúde Coletiva, v. 11, n. 4, p. 863-869, 2006.

WALDMAN, E. Inquéritos populacionais: aspectos metodológicos, operacionais e éticos. Revista Brasileira de Epidemiologia, v. 11, n. 3, p. 168-179, 2008.

WHO - World Health Organization. The International Classification of Functioning, Disability and Health. WHO, 2001.

\section{Sobre os autores}

Luciana Castaneda é mestre em Saúde Coletiva pelo Instituto de Estudos em Saúde Coletiva da Universidade Federal do Rio de Janeiro. Fisioterapeuta, professora do Instituto Brasileiro de Medicina de Reabilitação - Laureate Universities.

Shamyr Sulyvan de Castro é doutor em Saúde Pública pela Faculdade de Saúde Pública da Universidade de São Paulo. Fisioterapeuta, professor adjunto do Departamento de Fisioterapia Aplicada - Instituto de Ciências da Saúde (ICS) - Universidade Federal do Triângulo Mineiro (UFTM).

Ligia Bahia é doutora em Saúde Pública pela Escola Nacional de Saúde Pública da Fundação Oswaldo Cruz. Médica, professora adjunta do Instituto de Estudos em Saúde Coletiva da Universidade Federal do Rio de Janeiro.

\section{Endereço para correspondência}

Luciana Castaneda

Praia de Botafogo, 158

22220-001 - Rio de Janeiro-RJ, Brasil 


\begin{abstract}
Disability Constructs of the Brazilian National Household Survey (PNAD): An analysis based on the International Classification of Functioning, Disability and Health (ICF)

The objective of this paper is to identify content common to the (PNAD) and the International Classification of Functioning, Disability and Health (ICF). The study is methodologically descriptive. The first stage of the research consisted of the identification of the blocks present in the data collection instrument whose content was related to functioning, disability and health. Next the meaningful content of the blocks and modules were selected. Finally the meaningful contents were extracted and coded for the ICF categories. The most common domains were those related to activities and participation, followed by those of bodily functions. Only one content was related to environmental factors. The study showed that the ICF is a tool with wide applicability in information systems of primary data. Due to its universal language and biopsychosocial perspective, the ICF should be further explored in the field of epidemiology.
\end{abstract}

Keywords: Health surveys. International Classification of Functioning, Disability and Health. Data collection. Information systems. Epidemiology.

\title{
Resumen
}

Constructos sobre la discapacidad presentes en la Encuesta Nacional de Hogares de Brasil (PNAD): un análisis basado en la Clasificación Internacional del Funcionamiento, de la Discapacidad y de la Salud (CIF)

El objetivo de este trabajo es identificar los constructos relacionados con la discapacidad presentes en la Pesquisa Nacional por Amostra de Domicílios (PNAD), considerando como herramienta teórica la Clasificación Internacional del Funcionamiento, de la Discapacidad y de la Salud (CIF). Se trata de un estudio metodológico de tipo descriptivo. La primera etapa de la investigación comprendió la identificación de los módulos del instrumento que incluían contenidos referentes al funcionamiento, la discapacidad y la deficiencia. Posteriormente, los contenidos significativos fueron extraídos y codificados según las categorías de la CIF. Se encontró que los dominios más frecuentes eran los relacionados con las actividades y la participación, seguidos por aquellos vinculados con las funciones del cuerpo. Solo uno de los contenidos estaba relacionado con factores ambientales. El estudio mostró que la CIF es una herramienta con gran aplicabilidad en los sistemas de información de datos primarios. Por su lenguaje universal y la perspectiva biopsicosocial que adopta, la clasificación debe ser más explorada en el campo de la epidemiología.

Palabras clave: Encuestas epidemiológicas. Clasificación Internacional del Funcionamiento, de la Discapacidad y de la Salud. Recolección de datos. Sistemas de información; Epidemiología.

Recebido para publicação em 21/01/2013

Aceito para publicação em 03/09/2014 
